\title{
Exact solution for the distribution of transmission eigenvalues in a disordered wire and comparison with random-matrix theory
}

\author{
C. W. J. Beenakker and B. Rejaei \\ Instıtuut-Lorentz, Universıty of Leiden, P.O. Box 9506, 2300 RA Leiden, The Netherlands
}

(Received 28 October 1993)

\begin{abstract}
We consider the complete probability distribution $P\left(\left\{T_{n}\right\}\right)$ of the transmission eigenvalues $T_{1}, T_{2}, \ldots, T_{N}$ of a disordered quasi-one-dimensional conductor (length $L$ much greater than width $W$ and mean free path $l$ ). The Fokker-Planck equation which describes the evolution of $P$ with increasing $L$ is mapped onto a Schrödinger equation by a Sutherland-type transformation. In the absence of time-reversal symmetry (e.g., because of a magnetic field), the mapping is onto a freefermion problem, which we solve exactly. The resulting distribution is compared with the predictions of random-matrix theory (RMT) in the metallic regime $(L \ll N l)$ and in the insulating regime $(L \gg N l)$. We find that the logarithmic eigenvalue repulsion of RMT is exact for $T_{n}$ 's close to unity, but overestimates the repulsion for weakly transmitting channels. The nonlogarithmic repulsion resolves several long-standing discrepancies between RMT and microscopic theory, notably in the magnitude of the universal conductance fluctuations in the metallic regime, and in the width of the log-normal conductance distribution in the insulating regime.
\end{abstract}

\section{INTRODUCTION}

A fundamental problem of mesoscopic physics is to find the statistical distribution of the scattering matrix in an ensemble of disordered conductors. Once this is known, one can compute all moments of the conductance, and of any other transport property, at temperatures which are sufficiently low that the conductor is fully phase coherent. Random-matrix theory (RMT) addresses this problem on the basis of the assumption that all correlations between the transmission eigenvalues are due to the $\mathrm{Ja}$ cobian from matrix to eigenvalue space. ${ }^{1-3}$ The transmission eigenvalues $T_{1}, T_{2}, \ldots, T_{N}$ are the eigenvalues of the matrix product $t t^{\dagger}$, where $t$ is the $N \times N$ transmission matrix of the conductor. The Jacobian is

$$
J\left(\left\{\lambda_{n}\right\}\right)=\prod_{i<\jmath}\left|\lambda_{\jmath}-\lambda_{\imath}\right|^{\beta},
$$

where $\lambda_{2} \equiv\left(1-T_{2}\right) / T_{2}$ is the ratio of reflection to transmission probabilities $(\lambda \geq 0$, since $0 \leq T \leq 1)$, and $\beta \in\{1,2,4\}$ is the symmetry index of the ensemble of scattering matrices. [In the absence of time-reversal symmetry, one has $\beta=2$; in the presence of time-reversal symmetry, one has $\beta=1(4)$ in the presence (absence) of spin-rotation symmetry.]

If all correlations are due to the Jacobian, then the probability distribution $P\left(\lambda_{1}, \lambda_{2}, \ldots, \lambda_{N}\right)$ of the $\lambda$ 's should have the form $P \propto J \prod_{\imath} f\left(\lambda_{2}\right)$, or equivalently,

$$
\begin{aligned}
& P\left(\left\{\lambda_{n}\right\}\right)=C \exp \left[-\beta\left(\sum_{2<j} u\left(\lambda_{2}, \lambda_{\jmath}\right)+\sum_{2} V\left(\lambda_{2}\right)\right)\right], \\
& u\left(\lambda_{2}, \lambda_{\jmath}\right)=-\ln \left|\lambda_{\jmath}-\lambda_{2}\right|
\end{aligned}
$$

with $V=-\beta^{-1} \ln f$ and $C$ a normalization constant. Equation (1.2) has the form of a Gibbs distribution at temperature $\beta^{-1}$ for a fictitious system of classical particles moving in one dimension in an external potential $V$, with a logarithmically repulsive interaction $u$. All microscopic parameters (sample length $L$, width $W$, mean free path $l$, Fermi wavelength $\lambda_{\mathrm{F}}$ ) are contained in the single function $V(\lambda)$. The logarithmic repulsion is independent of microscopic parameters because of its geometric origin.

The RMT probability distribution (1.2), due to Muttalib, Pichard, and Stone, ${ }^{2}$ was justified by a maximumentropy principle for quasi-one-dimensional (quasi-1D) conductors. ${ }^{2,3}$ Quasi-1D means $L \gg W$. In this limit one can assume that the distribution of scattering matrices is only a function of the transmission eigenvalues (isotropy assumption). The distribution (1.2) then maximizes the information entropy subject to the constraint of a given density of eigenvalues. The function $V(\lambda)$ is determined by this constraint and is not specified by RMT.

It was initially believed that Eq. (1.2) would provide an exact description in the quasi-1D limit if only $V(\lambda)$ were suitably chosen. ${ }^{3,4}$ However, it was shown recently by one of us $^{5}$ that RMT is not exact, even in the quasi-1D limit. If one computes from Eq. (1.2) in the metallic regime the variance $\operatorname{Var} G$ of the conductance $G=G_{0} \sum_{n} T_{n}$ (with $\left.G_{0}=2 e^{2} / h\right)$, one finds ${ }^{5}$

$$
\operatorname{Var} G / G_{0}=\frac{1}{8} \beta^{-1},
$$

independent of the form of $V(\lambda)$. The diagrammatic perturbation theory ${ }^{6,7}$ of universal conductance fluctuations (UCF) gives instead

$$
\operatorname{Var} G / G_{0}=\frac{2}{15} \beta^{-1}
$$

for a quasi-1D conductor. The difference between the coefficients $\frac{1}{8}$ and $\frac{2}{15}$ is tiny, but it has the fundamental 
implication that the interaction between the $\lambda$ variables is not precisely logarithmic, or in other words, that there exist correlations between the transmission eigenvalues over and above those induced by the Jacobian.

What then is the status of the random-matrix theory of quantum transport? It is obviously highly accurate, so that the true eigenvalue interaction should be close to logarithmic. Is there perhaps a cutoff for large separation of the $\lambda$ 's? Or is the true interaction a many-body interaction which cannot be reduced to the sum of pairwise interactions? That is the problem addressed in this paper. A brief account of our results was reported recently. ${ }^{8}$

The transport problem considered here has a counterpart in equilibrium. The Wigner-Dyson RMT of the statistics of the eigenvalues $\left\{E_{n}\right\}$ of a random Hamiltonian yields a probability distribution of the form (1.2), with a logarithmic repulsion between the energy levels. ${ }^{9}$ It was shown by Efetov ${ }^{10}$ and by Al'tshuler and Shklovskii ${ }^{11}$ that the logarithmic level repulsion in a small disordered particle (diameter $L$, diffusion constant $D$ ) holds for energy separations small compared to the Thouless energy $E_{\mathrm{c}} \equiv \hbar D / L^{2}$. For larger separations the interaction potential decays algebraically. ${ }^{12}$ As we will see, the way in which the RMT of quantum transport breaks down is quite different: The interaction $u\left(\lambda_{i}, \lambda_{j}\right)=-\ln \left|\lambda_{j}-\lambda_{i}\right|$ is exact for $\lambda_{i}, \lambda_{j} \ll 1$, i.e., for strongly transmitting scattering channels [recall that $\lambda \ll 1$ implies $T \equiv(1+\lambda)^{-1}$ close to unity]. For weakly transmitting channels the repulsion is still logarithmic, but reduced by a factor of 2 from what one would expect from the Jacobian. This modified interaction explains the $\frac{1}{8}-\frac{2}{15}$ discrepancy in the UCF in the metallic regime, ${ }^{5}$ and it also explains a missing factor of 2 in the width of the log-normal distribution of the conductance in the insulating regime. ${ }^{13}$

Our analysis is based on the Dorokhov-Mello-PereyraKumar (DMPK) equation

$$
l \frac{\partial P}{\partial L}=\frac{2}{\beta N+2-\beta} \sum_{i=1}^{N} \frac{\partial}{\partial \lambda_{i}} \lambda_{i}\left(1+\lambda_{i}\right) J \frac{\partial}{\partial \lambda_{i}} J^{-1} P
$$

with ballistic initial condition $\lim _{L \rightarrow 0} P=\prod_{i} \delta\left(\lambda_{i}-0^{+}\right)$, which describes the evolution of the eigenvalue distribution function in an ensemble of disordered wires of increasing length. Equation (1.5) was derived by Dorokhov, ${ }^{14}$ (for $\beta=2$ ) and by Mello, Pereyra, and Kumar, ${ }^{15}$ (for $\beta=1$, with generalizations to $\beta=2,4$ in Refs. 16,17$)$ by computing the incremental change of the transmission eigenvalues upon attachment of a thin slice to the wire. It is assumed that the conductor is weakly disordered, $l \gg \lambda_{F}$, so that the scattering in the thin slice can be treated by perturbation theory. A key simplification is the isotropy assumption that the flux incident in one scattering channel is, on average, equally distributed among all outgoing channels. This assumption restricts the applicability of the DMPK equation to the quasi-1D regime $L \gg W$, since it ignores the finite time scale for transverse diffusion.
Equation (1.5) has the form of a diffusion equation in a complicated $N$-dimensional space (identified as a certain Riemannian manifold in Ref. 18). For a coordinatefree "supersymmetry formulation" of this diffusion process, see Refs. 19 and 20. The similarity to diffusion in real space has been given further substance by the demonstration ${ }^{21}$ that Eq. (1.5) holds on length scales $\gg l$ regardless of the microscopic scattering properties of the conductor.

The diffusion equation (1.5) has been studied extensively for more than ten years. Exact solutions have been obtained by Mel'nikov ${ }^{22}$ and Mello ${ }^{23}$ for the case $N=1$ of a single degree of freedom (when $J \equiv 1$ ). For $N>1$ the strong coupling of the scattering channels by the Jacobian (1.1) prevented an exact solution by standard methods. The problem simplifies drastically deep in the localized regime $(L \gg N l)$, when the scattering channels become effectively decoupled. Pichard ${ }^{13}$ has computed from Eq. (1.5) the log-normal distribution of the conductance in this regime, and has found excellent agreement with numerical simulations of a quasi-1D Anderson insulator. In the metallic regime $(L \ll N l)$, Mello and Stone ${ }^{16,24}$ were able to compute the first two moments of the conductance, in precise agreement with the diagrammatic perturbation theory of weak localization and UCF [Eq. (1.4)] in the quasi-1D limit. (Their method of moments has also been applied to the shot noise, ${ }^{25}$ where there is no diagrammatic theory to compare with.) More general calculations of the weak-localization effect ${ }^{26}$ and of universal fluctuations ${ }^{27}$ [for arbitrary transport properties of the form $A=\sum_{n} a\left(T_{n}\right)$ ] were recently developed, based on linearization of Eq. (1.5) in the fluctuations of the $\lambda$ 's around their mean positions (valid in the large- $N$ metallic regime, when the fluctuations are small). The work of Chalker and $\mathrm{Macêdo}^{27}$ was motivated by the same $\frac{1}{8}-\frac{2}{15}$ discrepancy ${ }^{5}$ as the present paper and Ref. 8, with which it has some overlap.

None of these calculations suffices to determine the form of the eigenvalue interaction, which requires knowledge of the complete distribution function. Here we wish to present (in considerable more detail than in our Ref. 8) the exact solution of Eq. (1.5) for $\beta=2$.

The outline of this paper is as follows. In Sec. II we solve Eq. (1.5) exactly, for all $N$ and $L$, for the case $\beta=2$. The method of solution is a mapping onto a model of noninteracting fermions, inspired by Sutherland's mapping of a different diffusion equation. ${ }^{28}$ The case $\beta=2$ is special, because for other values of $\beta$ the mapping introduces interactions between the fermions. The free-fermion problem, which is obtained for $\beta=2$, has the character of a one-dimensional scattering problem in imaginary time. The absence of a ground state is a significant complication, compared with Sutherland's problem. ${ }^{29-31}$ The exact solution which we obtain has the form of a determinant of an $N \times N$ matrix. The determinant can be evaluated in closed form in the metallic regime $L \ll N l$ and in the insulating regime $L \gg N l$. These two opposite regimes are discussed separately in Secs. III and IV. We conclude in Sec. V with a comparison of the solution of Eq. (1.5) with the probability distribution (1.2) of random-matrix theory. 


\section{EXACT SOLUTION}

The solution of the Dorokhov-Mello-Pereyra-Kumar equation (1.5) proceeds in a series of steps, which we describe in separate subsections.

\section{A. Transformation of variables}

The DMPK equation (1.5) can be written in the form of an $N$-dimensional Fokker-Planck equation,

$\frac{\partial}{\partial s} P\left(\left\{\lambda_{n}\right\}, s\right)=\sum_{\imath=1}^{N} \frac{\partial}{\partial \lambda_{\imath}} D\left(\lambda_{\imath}\right)\left(\frac{\partial P}{\partial \lambda_{\imath}}+\beta P \frac{\partial}{\partial \lambda_{\imath}} \Omega\left(\left\{\lambda_{n}\right\}\right)\right)$,

$D(\lambda)=\frac{2}{\gamma} \lambda(1+\lambda)$

$\Omega\left(\left\{\lambda_{n}\right\}\right)=-\sum_{2<j} \ln \left|\lambda_{3}-\lambda_{2}\right|$

where we have abbreviated $s=L / l, \gamma=\beta N+2-\beta$. Equation (2.1) is the diffusion equation in "time" $s$ of a one-dimensional gas of $N$ classical particles with a logarithmically repulsive interaction potential $\Omega$. The diffusion takes place at temperature $\beta^{-1}$ in a fictitious nonuniform viscous fluid with diffusion coefficient $D(\lambda)$.

The position dependence of the diffusion coefficient is problematic. We seek to eliminate it by a transformation of variables. Let $\left\{x_{n}\right\}$ be a new set of $N$ independent variables, related to the $\lambda$ 's by $\lambda_{n}=$ $f\left(x_{n}\right)$. The new probability distribution $P\left(\left\{x_{n}\right\}, s\right)=$ $P\left(\left\{\lambda_{n}\right\}, s\right) \prod_{2}\left|f^{\prime}\left(x_{2}\right)\right|$ still satisfies a Fokker-Planck equation, but with a new potential $\Omega\left(\left\{x_{n}\right\}\right)$ and a new diffusion coefficient $D(x)$. The potential transforms as $\Omega \rightarrow \Omega-\beta^{-1} \sum_{2} \ln \left|f^{\prime}\left(x_{2}\right)\right|$, while the diffusion coefficient transforms as $D \rightarrow D / f^{\prime}(x)^{2}$. In order to obtain an $x$ independent diffusion coefficient, we thus need to choose $f(x)$ such that $f(x)[1+f(x)] / f^{\prime}(x)^{2}=$ const. The choice $f(x)=\sinh ^{2} x$ does this.

We therefore transform to a new set of variables $\left\{x_{n}\right\}$, defined by

$$
\lambda_{n}=\sinh ^{2} x_{n}, T_{n}=1 / \cosh ^{2} x_{n} .
$$

Since $T_{n} \in[0,1], x_{n} \geq 0$. The probability distribution of the $x$ variables satisfies a Fokker-Planck equation with constant diffusion coefficient,

$$
\frac{\partial}{\partial s} P\left(\left\{x_{n}\right\}, s\right)=\frac{1}{2 \gamma} \sum_{\imath=1}^{N} \frac{\partial}{\partial x_{\imath}}\left(\frac{\partial P}{\partial x_{\imath}}+\beta P \frac{\partial}{\partial x_{\imath}} \Omega\left(\left\{x_{n}\right\}\right)\right),
$$

$$
\begin{aligned}
\Omega\left(\left\{x_{n}\right\}\right)= & -\sum_{2<j} \ln \left|\sinh ^{2} x_{J}-\sinh ^{2} x_{2}\right| \\
& -\frac{1}{\beta} \sum_{\imath} \ln \left|\sinh 2 x_{2}\right| .
\end{aligned}
$$

It turns out that the $x$ variables have a special physical significance: The ratio $L / x_{n}$ equals the channeldependent localization length of the conductor. ${ }^{3}$

\section{B. From the Fokker-Planck to Schrödinger equation}

Sutherland ${ }^{28}$ has shown that a Fokker-Planck equation with constant diffusion coefficient and with a logarithmic interaction potential can be mapped onto a Schrödinger equation with an inverse-square interaction which vanishes for $\beta=2$. The Fokker-Planck equation (2.3) does have a constant diffusion coefficient, but the interaction is not logarithmic. It is not obvious that Sutherland's mapping onto a free-fermion problem should work for the non-translationally-invariant interaction $(2.3 \mathrm{~b})$, but surprisingly enough it does.

To map the Fokker-Planck equation (2.3) onto a Schrödinger equation we substitute

$$
P\left(\left\{x_{n}\right\}, s\right)=\exp \left[-\frac{1}{2} \beta \Omega\left(\left\{x_{n}\right\}\right)\right] \Psi\left(\left\{x_{n}\right\}, s\right) .
$$

This is a variation on Sutherland's transformation, ${ }^{28}$ which we used in Ref. 31 in a different context. Substitution of Eq. (2.4) into Eq. (2.3a) yields for $\Psi$ the equation

$$
-\frac{\partial \Psi}{\partial s}=-\frac{1}{2 \gamma} \sum_{\imath=1}^{N} \frac{\partial^{2} \Psi}{\partial x_{\imath}^{2}}+\frac{\beta}{4 \gamma} \Psi \sum_{\imath=1}^{N}\left[\frac{\beta}{2}\left(\frac{\partial \Omega}{\partial x_{\imath}}\right)^{2}-\frac{\partial^{2} \Omega}{\partial x_{\imath}^{2}}\right] .
$$

The expression between square brackets is evaluated as follows (we abbreviate $\xi_{2}=\cosh 2 x_{\imath}$ ):

$$
\begin{aligned}
\sum_{\imath=1}^{N} \frac{\partial^{2} \Omega}{\partial x_{\imath}^{2}} & =4 \sum_{\imath} \sum_{\jmath(\neq \imath)}\left(\frac{\xi_{\imath}^{2}-1}{\left(\xi_{\jmath}-\xi_{\imath}\right)^{2}}+\frac{\xi_{\imath}}{\xi_{\jmath}-\xi_{\imath}}\right)+\frac{4}{\beta} \sum_{\imath} \frac{1}{\xi_{\imath}^{2}-1} \\
& =4 \sum_{\imath} \sum_{\jmath(\neq \imath)} \frac{\xi_{\imath}^{2}-1}{\left(\xi_{\jmath}-\xi_{\imath}\right)^{2}}+\frac{4}{\beta} \sum_{\imath} \frac{1}{\xi_{\imath}^{2}-1}-4\left(\begin{array}{c}
N \\
2
\end{array}\right), \\
\sum_{\imath=1}^{N}\left(\frac{\partial \Omega}{\partial x_{\imath}}\right)^{2} & =4 \sum_{\imath} \sum_{\jmath(\neq \imath)} \sum_{k(\neq \imath)} \frac{\xi_{\imath}^{2}-1}{\left(\xi_{\jmath}-\xi_{\imath}\right)\left(\xi_{k}-\xi_{\imath}\right)}-\frac{8}{\beta} \sum_{\imath} \sum_{\jmath(\neq \imath)} \frac{\xi_{\imath}}{\xi_{\jmath}-\xi_{\imath}}+\frac{4}{\beta^{2}} \sum_{\imath} \frac{\xi_{\imath}^{2}}{\xi_{\imath}^{2}-1} \\
& =4 \sum_{\imath} \sum_{\jmath(\neq \imath)} \frac{\xi_{\imath}^{2}-1}{\left(\xi_{\jmath}-\xi_{\imath}\right)^{2}}+\frac{4}{\beta^{2}} \sum_{\imath} \frac{1}{\xi_{\imath}^{2}-1}+\frac{4}{\beta^{2}} N+\frac{8}{\beta}\left(\begin{array}{c}
N \\
2
\end{array}\right)+8\left(\begin{array}{c}
N \\
3
\end{array}\right) .
\end{aligned}
$$


In the final equality we have used that for any three distinct indices $i, j, k$

$$
\begin{aligned}
& \frac{\xi_{\imath}^{2}-1}{\left(\xi_{\jmath}-\xi_{\imath}\right)\left(\xi_{k}-\xi_{\imath}\right)}+\frac{\xi_{\jmath}^{2}-1}{\left(\xi_{\imath}-\xi_{\jmath}\right)\left(\xi_{k}-\xi_{\jmath}\right)} \\
& +\frac{\xi_{k}^{2}-1}{\left(\xi_{\imath}-\xi_{k}\right)\left(\xi_{\jmath}-\xi_{k}\right)} \equiv 1,
\end{aligned}
$$

so that the triple sum over $k \neq \imath \neq \jmath$ collapses to a double sum over $\imath \neq j$. Collecting results, we find that $\Psi$ satisfies a Schrödinger equation in imaginary time,

$$
\begin{aligned}
-\frac{\partial \Psi}{\partial s}= & (\mathcal{H}-U) \Psi, \\
\mathcal{H}= & -\frac{1}{2 \gamma} \sum_{\imath}\left(\frac{\partial^{2}}{\partial x_{\imath}^{2}}+\frac{1}{\sinh ^{2} 2 x_{\imath}}\right) \\
& +\frac{\beta(\beta-2)}{2 \gamma} \sum_{\imath<\jmath} \frac{\sinh ^{2} 2 x_{\jmath}+\sinh ^{2} 2 x_{\imath}}{\left(\cosh 2 x_{\jmath}-\cosh 2 x_{\imath}\right)^{2}}, \\
U= & -\frac{N}{2 \gamma}-N(N-1) \frac{\beta}{\gamma}-N(N-1)(N-2) \frac{\beta^{2}}{6 \gamma} .
\end{aligned}
$$

The interaction potential in the Hamiltonian $(2.9 \mathrm{~b})$ is attractive for $\beta=1$ and repulsive for $\beta=4$. For $\beta=2$ the interaction vanishes identically, reducing $\mathcal{H}$ to a sum of single-particle Hamiltonians $\mathcal{H}_{0}$,

$$
\mathcal{H}_{0}=-\frac{1}{4 N} \frac{\partial^{2}}{\partial x^{2}}-\frac{1}{4 N \sinh ^{2} 2 x} .
$$

(Note that $\gamma=2 N$ for $\beta=2$.) It might be possible to solve also the interacting Schrödinger equation (2.9) for $\beta=1$ or 4 by some modification of techniques developed for the Sutherland Hamiltonian, ${ }^{28-30}$ but in this paper we focus on the simplest case $\beta=2$ of broken time-reversal symmetry.

To complete the mapping onto a single-particle problem, we need to consider the boundary condition at the edge $x=0$. (Recall that $x \geq 0$.) Conservation of probability implies for $P$ the boundary condition (one for each $i=1,2, \ldots, N)$

$$
\lim _{x_{\imath} \rightarrow 0}\left(\frac{\partial P}{\partial x_{\imath}}+\beta P \frac{\partial \Omega}{\partial x_{\imath}}\right)=0 .
$$

According to Eq. (2.4), the corresponding boundary condition on $\Psi$ is

$$
\lim _{x_{2} \rightarrow 0}\left(\frac{\partial \Psi}{\partial x_{\imath}}+\frac{1}{2} \beta \Psi \frac{\partial \Omega}{\partial x_{\imath}}\right)=0
$$

which in view of Eq. (2.3b) simplifies to

$$
\lim _{x_{i} \rightarrow 0}\left(\frac{\partial \Psi}{\partial x_{\imath}}-\frac{\Psi}{\sinh 2 x_{\imath}}\right)=0,
$$

independent of $\beta$. Fortunately, the boundary condition does not couple different degrees of freedom, so that we have indeed obtained a single-particle problem for $\beta=2$.

\section{From the probability distribution to the fermion} Green's function

We seek a solution $P\left(\left\{x_{n}\right\}, s \mid\left\{y_{n}\right\}\right)$ of the FokkerPlanck equation (2.3) with symmetrized $\delta$-function initial condition

$$
P\left(\left\{x_{n}\right\}, 0 \mid\left\{y_{n}\right\}\right)=\frac{1}{N !} \sum_{\pi} \prod_{\imath=1}^{N} \delta\left(x_{\imath}-y_{\pi_{\imath}}\right) .
$$

The sum in Eq. (2.14) is over all $N$ ! permutations of $1,2, \ldots, N$. Eventually, we will take the limit $\left\{y_{n}\right\} \rightarrow 0$ of a ballistic initial condition, but it is convenient to first consider the more general initial condition (2.14). In this subsection we use the mapping onto a Schrödinger equation of the previous subsection to relate the probability distribution $P\left(\left\{x_{n}\right\}, s \mid\left\{y_{n}\right\}\right)$ to the $N$-fermion Green's function $G\left(\left\{x_{n}\right\}, s \mid\left\{y_{n}\right\}\right)$.

We first note that, since $\exp (-\beta \Omega)$ is an $s$-independent solution of the Fokker-Planck equation (2.3), $\exp \left(-\frac{1}{2} \beta \Omega\right)$ is an $s$-independent solution of the Schrödinger equation (2.9) [in view of the mapping (2.4)]. For a particular ordering of the $x_{n}$ 's, the function $\Psi_{0} \propto \exp \left(-\frac{1}{2} \beta \Omega\right)$ is therefore an eigenfunction of the $N$-fermion Hamiltonian $\mathcal{H}$ with eigenvalue $U$. Antisymmetrization yields the fermion eigenstate

$$
\Psi_{0}\left(\left\{x_{n}\right\}\right)=C \exp \left[-\frac{1}{2} \beta \Omega\left(\left\{x_{n}\right\}\right)\right] \prod_{\imath<j} \frac{x_{3}-x_{\imath}}{\left|x_{3}-x_{\imath}\right|},
$$

with $C$ a normalization constant.

We obtain the $N$-fermion Green's function $G$ from the probability distribution $P$ by the similarity transformation

$G\left(\left\{x_{n}\right\}, s \mid\left\{y_{n}\right\}\right)$

$$
=\Psi_{0}^{-1}\left(\left\{x_{n}\right\}\right) P\left(\left\{x_{n}\right\}, s \mid\left\{y_{n}\right\}\right) \Psi_{0}\left(\left\{y_{n}\right\}\right) .
$$

To verify this, we first observe that $G$ is by construction antisymmetric under a permutation of two $x$ or two $y$ variables. For a given order of the $x_{n}$ 's, the function $G$ satisfies the Schrödinger equation

$$
-\frac{\partial G}{\partial s}=(\mathcal{H}-U) G
$$

in view of Eqs, (2.4), (2.9a), and (2.15). Finally, Eq. (2.14) implies the initial condition

$$
G\left(\left\{x_{n}\right\}, 0 \mid\left\{y_{n}\right\}\right)=\frac{1}{N !} \sum_{\pi} \sigma_{\pi} \prod_{\imath=1}^{N} \delta\left(x_{\imath}-y_{\pi_{\imath}}\right),
$$

with $\sigma_{\pi}$ the sign of the permutation. Hence $G$ is indeed the $N$-fermion Green's function.

The relation (2.16) holds for any $\beta$. In the remainder 
of this paper we consider the noninteracting case $\beta=2$. The eigenstate (2.15) then takes the form

$\Psi_{0}\left(\left\{x_{n}\right\}\right)=C \prod_{i<j}\left(\sinh ^{2} x_{j}-\sinh ^{2} x_{i}\right) \prod_{i}\left(\sinh 2 x_{i}\right)^{1 / 2}$.

The $N$-fermion Green's function $G$ becomes a Slater determinant of the single-particle Green's function $G_{0}$,

$$
G\left(\left\{x_{n}\right\}, s \mid\left\{y_{n}\right\}\right)=\frac{\mathrm{e}^{U s}}{N !} \operatorname{Det} G_{0}\left(x_{n}, s \mid y_{m}\right),
$$

where Det $a_{n m}$ denotes the determinant of the $N \times N$ matrix with elements $a_{n m}$. The function $G_{0}(x, s \mid y)$ is a solution of the single-particle Schrödinger equation $-\partial G_{0} / \partial s=\mathcal{H}_{0} G_{0}$ in the variable $x$, with initial condition $G(x, 0 \mid y)=\delta(x-y)$. In the following subsection we will compute the single-particle Green's function $G_{0}$. The probability distribution $P$, for $\beta=2$, then follows from Eqs. (2.16), (2.19), and (2.20):

$$
P\left(\left\{x_{n}\right\}, s \mid\left\{y_{n}\right\}\right)=\frac{\prod_{i<j}\left(\sinh ^{2} x_{j}-\sinh ^{2} x_{i}\right) \prod_{i}\left(\sinh 2 x_{i}\right)^{1 / 2}}{\prod_{i<j}\left(\sinh ^{2} y_{j}-\sinh ^{2} y_{i}\right) \prod_{i}\left(\sinh 2 y_{i}\right)^{1 / 2}} \frac{\mathrm{e}^{U s}}{N !} \operatorname{Det} G_{0}\left(x_{n}, s \mid y_{m}\right) .
$$

\section{Computation of the Green's function}

To compute the Green's function $G_{0}$ of the singleparticle Hamiltonian (2.10) we need to solve the eigenvalue equation

$$
-\frac{1}{4 N} \frac{d^{2}}{d x^{2}} \psi(x)-\frac{1}{4 N} \frac{\psi(x)}{\sinh ^{2} 2 x}=\varepsilon \psi(x),
$$

with the boundary condition dictated by Eq. (2.13),

$$
\lim _{x \rightarrow 0}\left(\frac{d \psi}{d x}-\frac{\psi}{\sinh 2 x}\right)=0 .
$$

We have found that the substitution

$$
\psi(x)=(\sinh 2 x)^{1 / 2} f(\cosh 2 x)
$$

transforms Eq. (2.22) into Legendre's differential equation in the variable $z=\cosh 2 x$,

$$
\frac{d}{d z}\left[\left(1-z^{2}\right) \frac{d}{d z} f(z)\right]=\left(N \varepsilon+\frac{1}{4}\right) f(z) .
$$

The boundary condition (2.23) restricts the solutions of Eq. (2.25) to the Legendre functions of the first kind $\mathrm{P}_{\nu}(z)$. The index $\nu$ is given by $\nu=-\frac{1}{2}+\frac{1}{2} \mathrm{i} k$ with $k$ a real number. (These Legendre functions are also known as "toroidal functions," because they appear as solutions to the Laplace equation in toroidal coordinates.) The numbers $\nu, k$, and $\varepsilon$ are related by $-\nu(\nu+1)=N \varepsilon+\frac{1}{4}$ and $\varepsilon=\frac{1}{4} k^{2} / N$. We can restrict ourselves to $k \geq 0$, since the functions $\mathrm{P}_{-\frac{1}{2}+\frac{1}{2} i k}$ and $\mathrm{P}_{-\frac{1}{2}-\frac{1}{2} i k}$ are identical.

We conclude that the spectrum of $\mathcal{H}_{0}$ is continuous, with positive eigenvalues $\varepsilon=\frac{1}{4} k^{2} / N$. The eigenfunctions $\psi_{k}(x)$ are real functions given by

$$
\begin{aligned}
\psi_{k}(x)= & {\left[\pi k \tanh \left(\frac{1}{2} \pi k\right) \sinh (2 x)\right]^{1 / 2} } \\
& \times \mathrm{P}_{\frac{1}{2}(i k-1)}(\cosh 2 x)
\end{aligned}
$$

They form a complete and orthonormal set,

$$
\begin{aligned}
& \int_{0}^{\infty} d k \psi_{k}(x) \psi_{k}\left(x^{\prime}\right)=2 \pi \delta\left(x-x^{\prime}\right) \\
& \int_{0}^{\infty} d x \psi_{k}(x) \psi_{k^{\prime}}(x)=2 \pi \delta\left(k-k^{\prime}\right)
\end{aligned}
$$

in accordance with the inversion formula in Ref. 32. The single-particle Green's function $G_{0}$ has the corresponding spectral representation

$$
\begin{aligned}
G_{0}(x, s \mid y) & =(2 \pi)^{-1} \int_{0}^{\infty} d k \exp \left(-\frac{1}{4} k^{2} s / N\right) \psi_{k}(x) \psi_{k}(y) \\
& =\frac{1}{2}(\sinh 2 x \sinh 2 y)^{1 / 2} \int_{0}^{\infty} d k \exp \left(-\frac{1}{4} k^{2} s / N\right) k \tanh \left(\frac{1}{2} \pi k\right) \mathrm{P}_{\frac{1}{2}(i k-1)}(\cosh 2 x) \mathrm{P}_{\frac{1}{2}(i k-1)}(\cosh 2 y)
\end{aligned}
$$

\section{E. Ballistic initial condition}

Equations (2.21) and (2.29) together determine the probability distribution $P\left(\left\{x_{n}\right\}, s \mid\left\{y_{n}\right\}\right)$ with initial condition (2.14), 


$$
\begin{aligned}
P= & C(s) \frac{\prod_{\imath<j}\left(\sinh ^{2} x_{3}-\sinh ^{2} x_{\imath}\right) \prod_{\imath}\left(\sinh 2 x_{\imath}\right)}{\prod_{\imath<j}\left(\sinh ^{2} y_{3}-\sinh ^{2} y_{\imath}\right)} \\
& \times \operatorname{Det}\left[\int_{0}^{\infty} d k \exp \left(-\frac{1}{4} k^{2} s / N\right) k \tanh \left(\frac{1}{2} \pi k\right) \mathrm{P}_{\frac{1}{2}(\imath k-1)}\left(\cosh 2 x_{n}\right) \mathrm{P}_{\frac{1}{2}(\imath k-1)}\left(\cosh 2 y_{m}\right)\right] \\
= & C(s) \prod_{\imath<j}\left(\sinh ^{2} x_{3}-\sinh ^{2} x_{\imath}\right) \prod_{\imath}\left(\sinh 2 x_{\imath}\right) \int_{0}^{\infty} d k_{1} \int_{0}^{\infty} d k_{2} \cdots \int_{0}^{\infty} d k_{N} \\
& \times \prod_{\imath}\left[\exp \left(-\frac{1}{4} k_{\imath}^{2} s / N\right) k_{\imath} \tanh \left(\frac{1}{2} \pi k_{\imath}\right) \mathrm{P}_{\frac{1}{2}\left(\imath k_{\imath}-1\right)}\left(\cosh 2 x_{\imath}\right)\right] \frac{\operatorname{Det}_{\frac{1}{2}\left(\imath k_{n}-1\right)}\left(\cosh 2 y_{m}\right)}{\prod_{\imath<j}\left(\sinh ^{2} y_{3}-\sinh ^{2} y_{\imath}\right)}
\end{aligned}
$$

We have absorbed all $x$ and $y$ independent factors into the function $C(s)$, which is fixed by the requirement that $P$ is normalized to unity,

$$
\int_{0}^{\infty} d x_{1} \int_{0}^{\infty} d x_{2} \cdots \int_{0}^{\infty} d x_{N} P=1
$$

In the second equality in Eq. (2.30) we have applied the identity $\operatorname{Det}\left(b_{n} a_{n m}\right)=\left(\prod_{2} b_{\imath}\right) \operatorname{Det} a_{n m}$ to isolate the factors containing the $y$ variables.

Now it remains to take the limit $\left\{y_{n}\right\} \rightarrow 0$ of a ballistic initial condition. The limit is tricky because it involves a cancellation of zeros of the determinant in the numerator with zeros of the alternating function in the denominator. It is convenient to first write the alternating function as a Vandermonde determinant,

$$
\prod_{i<j}\left(\sinh ^{2} y_{\jmath}-\sinh ^{2} y_{\imath}\right)=\operatorname{Det}\left(\sinh ^{2} y_{m}\right)^{n-1} .
$$

Next, we expand the Legendre function in powers of $\sinh ^{2} y$,

$$
\mathrm{P}_{\frac{1}{2}(2 k-1)}(\cosh 2 y)=\sum_{p=1}^{\infty} c_{p}(k)\left(\sinh ^{2} y\right)^{p-1}
$$

The factors $c_{p}(k)$ are polynomials in $k^{2}$, with $c_{1}(k) \equiv 1$ and

$$
\begin{aligned}
c_{p}(k)= & (-1)^{p-1}\left[2^{p-1}(p-1) !\right]^{-2}\left(k^{2}+1^{2}\right) \\
& \times\left(k^{2}+3^{2}\right) \cdots\left[k^{2}+(2 p-3)^{2}\right]
\end{aligned}
$$

for $p \geq 2$. In the limit $y \rightarrow 0$, we can truncate the expansion (2.33) after the first $N$ terms, that is to say,

$$
\begin{aligned}
\lim _{\left\{y_{n}\right\} \rightarrow 0} \frac{\operatorname{Det} P_{\frac{1}{2}\left(2 k_{n}-1\right)}\left(\cosh 2 y_{m}\right)}{\prod_{\imath<j}\left(\sinh ^{2} y_{j}-\sinh ^{2} y_{\imath}\right)} \\
\quad=\lim _{\left\{y_{n}\right\} \rightarrow 0} \frac{\operatorname{Det}\left[\sum_{p=1}^{N} c_{p}\left(k_{n}\right)\left(\sinh ^{2} y_{m}\right)^{p-1}\right]}{\operatorname{Det}\left(\sinh ^{2} y_{m}\right)^{n-1}} .
\end{aligned}
$$

The numerator on the right-hand side of Eq. (2.35) factors as the product of two determinants, one of which is just the Vandermonde determinant in the denominator, so that the whole quotient reduces to the single determinant $\operatorname{Det} c_{m}\left(k_{n}\right)$. This determinant can be simplified by means of the identity

$$
\operatorname{Det} c_{m}\left(k_{n}\right)=c_{0} \operatorname{Det}\left(k_{n}^{2}\right)^{m-1},
$$

with $c_{0}$ a numerical coefficient. Equation (2.36) holds because the determinant of a matrix is unchanged if any one column of the matrix is added to any other column, so that we can reduce the polynomial $c_{m}(k)$ in $k^{2}$ of degree $m-1$ to just its highest order term $k^{2(m-1)}$ times a numerical coefficient.

Collecting results, we find

$$
\lim _{\left\{y_{n}\right\} \rightarrow 0} \frac{\operatorname{Det}_{\frac{1}{2}\left(\imath k_{n}-1\right)}\left(\cosh 2 y_{m}\right)}{\prod_{\imath<\jmath}\left(\sinh ^{2} y_{\jmath}-\sinh ^{2} y_{\imath}\right)}=c_{0} \operatorname{Det}\left(k_{n}^{2}\right)^{m-1} \text {. }
$$

Substituting into Eq. (2.30), and absorbing the coefficient $c_{0}$ in the function $C(s)$, we obtain the probability distribution $P\left(\left\{x_{n}\right\}, s\right)$ for a ballistic initial condition,

$$
\begin{aligned}
P\left(\left\{x_{n}\right\}, s\right)= & C(s) \prod_{\imath<\jmath}\left(\sinh ^{2} x_{\mathfrak{J}}-\sinh ^{2} x_{\imath}\right) \prod_{\imath}\left(\sinh 2 x_{\imath}\right) \\
& \times \operatorname{Det}\left[\int_{0}^{\infty} d k \exp \left(-\frac{1}{4} k^{2} s / N\right) \tanh \left(\frac{1}{2} \pi k\right) k^{2 m-1} \mathrm{P}_{\frac{1}{2}(2 k-1)}\left(\cosh 2 x_{n}\right)\right] .
\end{aligned}
$$


This is the exact solution of the DMPK equation for the case $\beta=2$.

\section{METALLIC REGIME}

\section{A. Probability distribution}

The solution (2.38) holds for any $s$ and $N$. It can be simplified in the regime $1 \ll s \ll N$ of a conductor which is long compared to the mean free path $l$ but short compared to the localization length $\mathrm{Nl}$. This is the metallic regime. The dominant contribution to the integral over $k$ in Eq. (2.38) then comes from the range $k \gtrsim(N / s)^{1 / 2} \gg 1$. In this range $\tanh \left(\frac{1}{2} \pi k\right) \rightarrow 1$ and the Legendre function simplifies to a Bessel function, ${ }^{33}$

$\mathrm{P}_{\frac{1}{2}(2 k-1)}(\cosh 2 x)=J_{0}(k x)\left(\frac{2 x}{\sinh 2 x}\right)^{\frac{1}{2}}$

for $k \gg 1$. (3.1)

The $k$ integration can now be carried out analytically,

$$
\begin{aligned}
\int_{0}^{\infty} d k & \exp \left(-\frac{1}{4} k^{2} s / N\right) k^{2 m-1} J_{0}\left(k x_{n}\right) \\
\quad= & \frac{1}{2}(m-1) !(4 N / s)^{m} \exp \left(-x_{n}^{2} N / s\right) L_{m-1}\left(x_{n}^{2} N / s\right),
\end{aligned}
$$

with $L_{m-1}$ a Laguerre polynomial. We then apply the determinantal identity

$$
\operatorname{Det} L_{m-1}\left(x_{n}^{2} N / s\right)=c \operatorname{Det}\left(x_{n}^{2}\right)^{m-1}=c \prod_{\imath<\jmath}\left(x_{\jmath}^{2}-x_{\imath}^{2}\right),
$$

with $c$ an $x$-independent number [which can be absorbed in $C(s)$ ]. Equation (3.3) is derived in the same way as Eq. (2.36), by combining columns of the matrix of polynomials in $x^{2}$. Collecting results, we find that the general solution (2.38) simplifies in the metallic regime to

$$
\begin{aligned}
P\left(\left\{x_{n}\right\}, s\right)= & C(s) \prod_{\imath<\jmath}\left[\left(\sinh ^{2} x_{3}-\sinh ^{2} x_{\imath}\right)\left(x_{\jmath}^{2}-x_{\imath}^{2}\right)\right] \\
& \times \prod_{\imath}\left[\exp \left(-x_{\imath}^{2} N / s\right)\left(x_{\imath} \sinh 2 x_{\imath}\right)^{1 / 2}\right] .
\end{aligned}
$$

In the remainder of this section we use the probability distribution (3.4) to compute various statistical quantities of interest. For that purpose it is convenient to write $P$ as a Gibbs distribution,

$$
\begin{aligned}
P\left(\left\{x_{n}\right\}, s\right)= & C(s) \exp \left[-\beta\left(\sum_{\imath<\jmath} u\left(x_{\imath}, x_{\jmath}\right)\right.\right. \\
& \left.\left.+\sum_{\imath} V\left(x_{\imath}, s\right)\right)\right] \\
u\left(x_{\imath}, x_{\jmath}\right)= & -\frac{1}{2} \ln \left|\sinh ^{2} x_{\jmath}-\sinh ^{2} x_{\imath}\right|-\frac{1}{2} \ln \left|x_{\jmath}^{2}-x_{\imath}^{2}\right|, \\
V(x, s)= & \frac{1}{2} N s^{-1} x^{2}-\frac{1}{4} \ln (x \sinh 2 x),
\end{aligned}
$$

with $\beta=2$ understood.

\section{B. Eigenvalue density}

The mean density $\langle\rho(x)\rangle_{s}$ of the $x$ variables is defined as the ensemble average with distribution $P\left(\left\{x_{n}\right\}, s\right)$ of the microscopic density $\rho(x)$ :

$$
\begin{aligned}
& \rho(x)=\sum_{n=1}^{N} \delta\left(x-x_{n}\right) \\
& \langle\rho(x)\rangle_{s}=\int_{0}^{\infty} d x_{1} \int_{0}^{\infty} d x_{2} \cdots \int_{0}^{\infty} d x_{N} P\left(\left\{x_{n}\right\}, s\right) \rho(x) .
\end{aligned}
$$

The mean density is determined to leading order in $N$ by the integral equation

$$
-\int_{0}^{\infty} d x^{\prime}\left\langle\rho\left(x^{\prime}\right)\right\rangle_{s} u\left(x, x^{\prime}\right)=V(x, s)+\text { const } .
$$

The additive constant (which may depend on $s$ but is independent of $x$ ) is fixed by the normalization condition

$$
\int_{0}^{\infty} d x\langle\rho(x)\rangle_{s}=N
$$

Equation (3.8) can be understood intuitively as the condition for mechanical equilibrium of a fictitious onedimensional gas with two-body interaction $u$ in a confining potential $V$. Dyson ${ }^{34}$ has shown that corrections to $\mathrm{Eq}$. (3.8) are an order $N^{-1} \ln N$ smaller than the terms retained, and are $\beta$ dependent. These corrections are responsible for the weak-localization effect in the conductance. ${ }^{26}$ Here we consider only the leadingorder contribution to the density, which is of order $N$ and which is independent of $\beta$.

Substituting the functions $u\left(x, x^{\prime}\right)$ and $V(x, s)$ from Eq. (3.5) into Eq. (3.8), and taking the derivative with respect to $x$ to eliminate the additive constant, we obtain the equation

$$
\begin{array}{r}
\frac{s}{2 N} \int_{0}^{\infty} d x^{\prime}\left\langle\rho\left(x^{\prime}\right)\right\rangle_{s}\left(\frac{\sinh 2 x}{\sinh ^{2} x-\sinh ^{2} x^{\prime}}+\frac{2 x}{x^{2}-x^{\prime 2}}\right) \\
=x+O(1 / N) .
\end{array}
$$

We note that

$$
\begin{aligned}
& \int_{0}^{s} d x^{\prime}\left(\frac{\sinh 2 x}{\sinh ^{2} x-\sinh ^{2} x^{\prime}}+\frac{2 x}{x^{2}-x^{\prime 2}}\right) \\
& =\ln \left|\frac{\sinh (s+x)}{\sinh (s-x)}\right|+\ln \left|\frac{s+x}{s-x}\right| \\
& =2 x+O(x / s) \text { for } s \gg 1, s \gg x \text {. }
\end{aligned}
$$

It follows that the uniform density

$$
\langle\rho(x)\rangle_{s}=\frac{N}{s} \theta(s-x)
$$


[The function $\theta(\xi)$ equals 1 for $\xi>0$ and 0 for $\xi<0$.] The result (3.12) was first obtained by Mello and Pichard, by direct integration of the DMPK equation. ${ }^{4}$ To order $N$, the $x$ variables have a uniform density of $N l / L$, with a cutoff at $L / l$ such that the normalization (3.9) is satisfied. In the cutoff region $x \sim L / l$ the density deviates from uniformity, but this region is irrelevant since the transmission eigenvalues are exponentially small for $x \gg 1$.

\section{Correlation function}

The two-point correlation function $K\left(x, x^{\prime}, s\right)$ is defined by

$$
K\left(x, x^{\prime}, s\right)=\langle\rho(x)\rangle_{s}\left\langle\rho\left(x^{\prime}\right)\right\rangle_{s}-\left\langle\rho(x) \rho\left(x^{\prime}\right)\right\rangle_{s} .
$$

We compute the two-point correlation function by the general method of Ref. 5, which is based on an exact relationship between $K$ and the functional derivative of the mean eigenvalue density $\langle\rho\rangle$ with respect to the eigenvalue potential $V$ :

$$
K\left(x, x^{\prime}, s\right)=\frac{1}{\beta} \frac{\delta\langle\rho(x)\rangle_{s}}{\delta V\left(x^{\prime}, s\right)} .
$$

Equation (3.14) holds for any probability distribution of the form (3.5a), regardless of whether the interaction is logarithmic or not. In the large- $N$ limit the functional derivative can be evaluated from the integral equation $(3.8)$. The functional derivative $\delta\langle\rho\rangle / \delta V$ equals the solving kernel of

$$
-\int_{0}^{\infty} d x^{\prime} \psi\left(x^{\prime}\right) u\left(x, x^{\prime}\right)=\phi(x)+\text { const }
$$

where the additive constant has to be chosen such that $\psi$ has zero mean,

$$
\int_{0}^{\infty} d x \psi(x)=0
$$

since the variations in $\langle\rho\rangle$ have to occur at constant $N$. Because of Eq. (3.14), the integral solution

$$
\psi(x)=\int_{0}^{\infty} d x^{\prime} \beta K\left(x, x^{\prime}\right) \phi\left(x^{\prime}\right)
$$

of Eq. (3.15) directly determines the two-point correlation function. It turns out that $K\left(x, x^{\prime}\right) \equiv K\left(x, x^{\prime}, s\right)$ is independent of $s$ in the metallic regime.

The integral equation (3.15) can be solved analytically by the following method. We extend the functions $\psi$ and $\phi$ symmetrically to negative $x$, by defining $\psi(-x) \equiv \psi(x), \phi(-x) \equiv \phi(x)$. We then note that the decomposition

$$
\begin{aligned}
u\left(x, x^{\prime}\right) & =-\frac{1}{2} \mathcal{U}\left(x-x^{\prime}\right)-\frac{1}{2} \mathcal{U}\left(x+x^{\prime}\right)+\ln 2 \\
\mathcal{U}(x) & =\ln |2 x \sinh x|
\end{aligned}
$$

transforms the integral equation (3.15) into a convolution,

$$
\int_{-\infty}^{\infty} d x^{\prime} \psi\left(x^{\prime}\right) \mathcal{U}\left(x-x^{\prime}\right)=2 \phi(x)+\text { const }
$$

which is readily solved by Fourier transformation. The Fourier-transformed kernel is

$$
\mathcal{U}(k) \equiv \int_{-\infty}^{\infty} d x \mathrm{e}^{i k x} \mathcal{U}(x)=-\frac{\pi}{|k|}\left[1+\operatorname{cotanh}\left(\frac{1}{2} \pi|k|\right)\right]
$$

The $k$-space solution to Eq. (3.19) is $\psi(k)=2 \phi(k) / \mathcal{U}(k)$, which automatically satisfies the normalization (3.16). In $x$ space the solution becomes

$$
\begin{aligned}
\psi(x) & =2 \int_{-\infty}^{\infty} d x^{\prime} \mathcal{K}\left(x-x^{\prime}\right) \phi\left(x^{\prime}\right) \\
& =2 \int_{0}^{\infty} d x^{\prime}\left[\mathcal{K}\left(x-x^{\prime}\right)+\mathcal{K}\left(x+x^{\prime}\right)\right] \phi\left(x^{\prime}\right) \\
\mathcal{K}(x) & =\frac{1}{2 \pi} \int_{-\infty}^{\infty} d k \mathrm{e}^{-i k x} \frac{1}{\mathcal{U}(k)}=\frac{1}{\pi} \int_{0}^{\infty} d k \frac{\cos k x}{\mathcal{U}(k)}
\end{aligned}
$$

Combining Eqs. (3.17), (3.20), and (3.21), we find that the two-point correlation function is given by

$$
\begin{aligned}
K\left(x, x^{\prime}\right) & =\mathcal{K}\left(x-x^{\prime}\right)+\mathcal{K}\left(x+x^{\prime}\right), \\
\mathcal{K}(x) & =-\frac{2}{\beta \pi^{2}} \int_{0}^{\infty} d k \frac{k \cos k x}{1+\operatorname{cotanh}\left(\frac{1}{2} \pi k\right)},
\end{aligned}
$$

with $\beta=2$. The inverse Fourier transform (3.22b) evaluates to

$$
\begin{aligned}
\mathcal{K}(x) & =\frac{1}{2 \beta \pi^{2}} \frac{d^{2}}{d x^{2}} \ln \left[1+(\pi / x)^{2}\right] \\
& =\frac{1}{\beta \pi^{2}} \operatorname{Re}\left[\left(x+i 0^{+}\right)^{-2}-(x+i \pi)^{-2}\right],
\end{aligned}
$$

where $0^{+}$is a positive infinitesimal.

We derived ${ }^{8}$ these expressions for the two-point correlation function for the case $\beta=2$. A direct integration of the DMPK equation by Chalker and Macêdo ${ }^{27}$ shows that the function $K\left(x, x^{\prime}\right)$ has in fact the $1 / \beta$ dependence indicated in Eq. (3.22), as expected from general considerations. ${ }^{5}$

\section{Universal conductance fluctuations}

Now that we have the two-point correlation function, we can compute the variance $\operatorname{Var} A=\left\langle A^{2}\right\rangle-\langle A\rangle^{2}$ of any linear statistic $A=\sum_{n=1}^{N} a\left(x_{n}\right)$ on the transmission eigenvalues (recall that $T_{n} \equiv \cosh ^{-2} x_{n}$ ). By definition

$$
\operatorname{Var} A=-\int_{0}^{\infty} d x \int_{0}^{\infty} d x^{\prime} a(x) a\left(x^{\prime}\right) K\left(x, x^{\prime}\right)
$$


Substituting Eq. (3.22) we find

$$
\begin{aligned}
& \operatorname{Var} A=\frac{1}{\beta \pi^{2}} \int_{0}^{\infty} d k \frac{k|a(k)|^{2}}{1+\operatorname{cotanh}\left(\frac{1}{2} \pi k\right)}, \\
& a(k)=2 \int_{0}^{\infty} d x a(x) \cos k x
\end{aligned}
$$

or equivalently,

$$
\begin{aligned}
\operatorname{Var} A= & -\frac{1}{2 \beta \pi^{2}} \int_{0}^{\infty} d x \int_{0}^{\infty} d x^{\prime}\left(\frac{d a(x)}{d x}\right)\left(\frac{d a\left(x^{\prime}\right)}{d x^{\prime}}\right) \\
& \times \ln \left(\frac{1+\pi^{2}\left(x+x^{\prime}\right)^{-2}}{1+\pi^{2}\left(x-x^{\prime}\right)^{-2}}\right)
\end{aligned}
$$

To obtain the variance of the conductance $G / G_{0}=$ $\sum_{n} T_{n}$ (with $G_{0}=2 e^{2} / h$ ), we substitute $a(x)=$ $\cosh ^{-2} x$, hence $a(k)=\pi k / \sinh \left(\frac{1}{2} \pi k\right)$, hence

$$
\operatorname{Var} G / G_{0}=\frac{2}{15} \beta^{-1} \text {, }
$$

in agreement with Eq. (1.4). In the same way one can compute the variance of other transport properties. For example, for the shot-noise power ${ }^{35} P / P_{0}=$ $\sum_{n} T_{n}\left(1-T_{n}\right.$ ) (with $P_{0}=2 e|V| G_{0}$ and $V$ the applied voltage) we substitute $a(x)=\cosh ^{-2} x-\cosh ^{-4} x$, hence $a(k)=\frac{1}{6} \pi k\left(2-k^{2}\right)\left(\sinh \frac{1}{2} \pi k\right)^{-1}$, hence

$$
\operatorname{Var} P / P_{0}=\frac{46}{2835} \beta^{-1}
$$

in agreement with the result obtained by a moment expansion of the DMPK equation. ${ }^{25}$ Another example is the conductance $G_{\text {NS }}$ of a normal-superconductor junction, which for $\beta=1$ is a linear statistic, ${ }^{36}$ $G_{\mathrm{NS}} / G_{0}=\sum_{n} 2 T_{n}^{2}\left(2-T_{n}\right)^{-2}$. We substitute $a(x)=$ $2 \cosh ^{-4} x\left(2-\cosh ^{-2} x\right)^{2}=2 \cosh ^{-2}(2 x)$, hence $a(k)=$ $\frac{1}{2} \pi k / \sinh \left(\frac{1}{4} \pi k\right)$, hence

$$
\operatorname{Var} G_{N S} / G_{0}=\frac{16}{15}-\frac{48}{\pi^{4}}
$$

Finally, for the variance of the critical current $I_{\mathrm{c}}$ of a point-contact Josephson junction (which is also a linear statistic for $\beta=1)^{37,38}$ we compute

$$
\operatorname{Var} I_{c} / I_{0}=0.0890,
$$

with $I_{0}=e \Delta / \hbar$ and $\Delta$ the superconducting energy gap.

As in the previous subsection, we note that our results are derived for $\beta=2$, and that the $1 / \beta$ dependence of the variance in Eq. (3.24) needs the justification provided by the calculation of Chalker and Macêdo. ${ }^{27}$

\section{INSULATING REGIME}

The solution (2.38) can also be simplified in the regime $1 \ll N \ll s$ of a conductor which is long compared to the localization length $\mathrm{Nl}$. This is the insulating regime. It is sufficient to consider the range $x_{n} \gg 1$, since the probability that $x \lesssim 1$ is of order $N / s$ which is $\ll 1$. The appropriate asymptotic expansion of the Legendre function is

$$
\begin{aligned}
P_{\frac{1}{2}(\imath k-1)}(\cosh 2 x)= & (2 \pi \sinh 2 x)^{-1 / 2} \\
& \times \operatorname{Re} \frac{2 \Gamma\left(\frac{1}{2} \imath k\right) \mathrm{e}^{\imath k x}}{\Gamma\left(\frac{1}{2}+\frac{1}{2} \imath k\right)} \text { for } x \gg 1 .
\end{aligned}
$$

For $s / N \gg 1$, the dominant contribution to the integral over $k$ in Eq. (2.38) comes from the range $k \ll 1$. In this range $\tanh \left(\frac{1}{2} \pi k\right) \rightarrow \frac{1}{2} \pi k$ and the ratio of Gamma functions in Eq. (4.1) simplifies to

$$
\frac{\Gamma\left(\frac{1}{2} \imath k\right)}{\Gamma\left(\frac{1}{2}+\frac{1}{2} i k\right)}=\left(\frac{1}{2} i k \sqrt{ } \pi\right)^{-1} \text { for } k \ll 1
$$

The $k$ integration can now be carried out analytically,

$$
\int_{0}^{\infty} d k \exp \left(-\frac{1}{4} k^{2} s / N\right) k^{2 m-1} \sin \left(k x_{n}\right)=(-1)^{m-1} \pi^{1 / 2}(N / s)^{m} \exp \left(-x_{n}^{2} N / s\right) H_{2 m-1}\left(x_{n} \sqrt{N / s}\right)
$$

with $H_{2 m-1}$ a Hermite polynomial. We then apply the determinantal identity [cf. Eq. (3.3)]

$$
\begin{aligned}
\operatorname{Det} H_{2 m-1}\left(x_{n} \sqrt{N / s}\right) & =c \operatorname{Det} x_{n}^{2 m-1} \\
& =c \prod_{\imath} x_{\imath} \prod_{\imath<\jmath}\left(x_{\jmath}^{2}-x_{\imath}^{2}\right)
\end{aligned}
$$

with $c$ an $x$-independent number. Collecting results, we find that the general solution (2.38) reduces in the insulating regime to

$$
\begin{aligned}
P\left(\left\{x_{n}\right\}, s\right)= & C(s) \prod_{\imath<j}\left[\left(\sinh ^{2} x_{\jmath}-\sinh ^{2} x_{\imath}\right)\left(x_{\jmath}^{2}-x_{\imath}^{2}\right)\right] \\
& \times \prod_{\imath}\left[\exp \left(-x_{\imath}^{2} N / s\right) x_{\imath}\left(\sinh 2 x_{\imath}\right)^{1 / 2}\right] \cdot
\end{aligned}
$$

(This formula was cited incorrectly in Ref. 8.)

The result (4.5) can be simplified further by ordering the $x_{n}$ 's from small to large and using the fact that $1 \ll$ $x_{1} \ll x_{2} \ll \cdots \ll x_{N}$ in the insulating regime $(s \gg N)$. The distribution function then factorizes, 


$$
\begin{aligned}
P\left(\left\{x_{n}\right\}, s\right) & =C(s) \prod_{\imath=1}^{N} \exp \left[(2 \imath-1) x_{\imath}-x_{\imath}^{2} N / s\right] \\
& =(\pi s / N)^{-N / 2} \prod_{\imath=1}^{N} \exp \left[-(N / s)\left(x_{\imath}-\bar{x}_{\imath}\right)^{2}\right]
\end{aligned}
$$

The $x_{n}$ 's have a Gaussian distribution with mean $\bar{x}_{n}=$ $\frac{1}{2}(s / N)(2 n-1)$ and variance $\frac{1}{2} s / N$. The width of the Gaussian is smaller than the mean spacing by a factor $(N / s)^{1 / 2}$, which is $\ll 1$, so that indeed $1 \ll x_{1} \ll x_{2} \ll$ $\cdots \ll x_{N}$, as anticipated.

The conductance $G / G_{0}=\sum_{n} \cosh ^{-2} x_{n}$ is dominated by $x_{1}$, i.e., by the smallest of the $x_{n}$ 's. Since $x_{1} \gg 1$ we may approximate $G / G_{0}=4 \exp \left(-2 x_{1}\right)$. It follows that the conductance has a log-normal distribution, with mean $\left\langle\ln G / G_{0}\right\rangle=-s / N+O(1)$ and variance $\operatorname{Var} \ln G / G_{0}=2 s / N$. Hence we conclude that

$$
\operatorname{Var} \ln G / G_{0}=-2\left\langle\ln G / G_{0}\right\rangle
$$

in agreement with the result obtained by Pichard, ${ }^{13}$ by directly solving the DMPK equation in the localized regime.

The results obtained here are for the case $\beta=2$. Pichard has shown that the relationship (4.7) between mean and variance of $\ln G / G_{0}$ remains valid for other values of $\beta$, since both the mean and the variance have a $1 / \beta$ dependence on the symmetry index.

\section{COMPARISON WITH RANDOM-MATRIX THEORY}

The random-matrix theory of quantum transport ${ }^{2,3}$ is based on the postulate that all correlations between the transmission eigenvalues are due to the Jacobian (1.1). The resulting distribution function (1.2) has the form of a Gibbs distribution with a logarithmic repulsive interaction in the variables $\lambda_{n} \equiv\left(1-T_{n}\right) / T_{n}$. There exists a maximum-entropy argument for this distribution, ${ }^{2,3}$ but it has no microscopic justification. In this paper we have shown, for the case of a quasi-1D geometry without timereversal symmetry, that the prediction of RMT is highly accurate but not exact.

In the metallic regime $(L \ll N l)$, the distribution is given by Eq. (3.5). In terms of the $\lambda$ variables $(\lambda \equiv$ $\sinh ^{2} x$ ), the distribution takes the form (1.2a) of RMT, but with a different interaction

$$
\begin{aligned}
u\left(\lambda_{2}, \lambda_{\jmath}\right)=-\frac{1}{2} \ln \left|\lambda_{J}-\lambda_{2}\right|-\frac{1}{2} \ln \mid \operatorname{arcsinh}^{2} \lambda_{\jmath}^{1 / 2} & \\
& -\operatorname{arcsinh}^{2} \lambda_{2}^{1 / 2} \mid .
\end{aligned}
$$

For $\lambda \ll 1$ (i.e., for $T$ close to unity) $u\left(\lambda_{2}, \lambda_{\jmath}\right) \rightarrow$ $-\ln \left|\lambda_{3}-\lambda_{2}\right|$, so we derive the logarithmic eigenvalue repulsion (1.2b) for the strongly transmitting scattering channels. However, for $\lambda \approx 1$ the interaction (5.1) is nonlogarnthmic. For fixed $\lambda_{2} \ll 1, u\left(\lambda_{2}, \lambda_{3}\right)$ as a function of $\lambda_{3}$ crosses over from $-\ln \left|\lambda_{3}-\lambda_{\imath}\right|$ to $-\frac{1}{2} \ln \left|\lambda_{j}-\lambda_{2}\right|$ as $\lambda_{j} \rightarrow \infty$ (see Fig. 1). It is remarkable that, for weakly

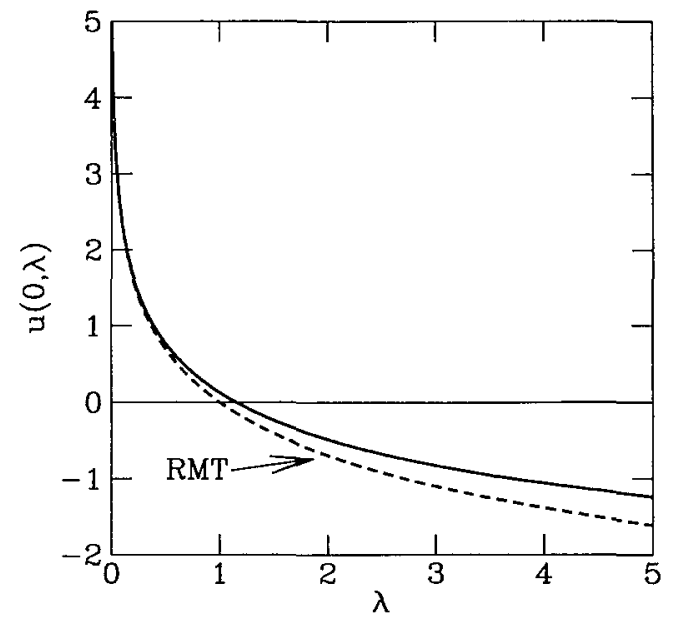

FIG. 1. Interaction potential $u\left(\lambda_{2}, \lambda_{\jmath}\right)$ for $\lambda_{2}=0$ as a function of $\lambda_{3} \equiv \lambda$. The solid curve is the result (5.1) from the DMPK equation. The dashed curve is the logarithmic repulsion (1.2b) predicted by random-matrix theory. For $\lambda \ll 1$ the two curves coincide For $\lambda \rightarrow \infty$ their ratio approaches a factor of 2 .

transmitting channels, the interaction is twice as small as predicted by considerations based solely on the Jacobian. We have no intuitive argument for this result. The reduced level repulsion for weakly transmitting channels enhances the variance of the conductance fluctuations above the prediction (1.3) of RMT. Indeed, as shown in Sec. IIID, a calculation along the lines of Ref. 5 , but for the nonlogarithmic interaction (5.1), resolves the $\frac{1}{8}-\frac{2}{15}$ discrepancy between RMT and diagrammatic perturbation theory, discussed in the Introduction. The discrepancy is so small because only the weakly transmitting channels (which contribute little to the conductance) are affected by the nonlogarithmic interaction.

In the insulating regime $(L \gg N l)$, the distribution is given by Eq. (4.5). In terms of the $\lambda$ 's the distribution takes the form (1.2a) of RMT, but again with the nonlogarithmic interaction (5.1). Since $\ln \lambda \gg 1$ in the insulating regime, the interaction (5.1) may be effectively simplified to $u\left(\lambda_{2}, \lambda_{\jmath}\right)=-\frac{1}{2} \ln \left|\lambda_{J}-\lambda_{2}\right|$, which is a factor of 2 smaller than the interaction (1.2b) predicted by RMT. This explains the factor-of-2 discrepancy between the results of RMT and of numerical simulations for the width of the log-normal distribution of the conductance: ${ }^{13}$ RMT predicts Var $\ln G / G_{0}=-\left\langle\ln G / G_{0}\right\rangle$, which is twice as small as the correct result (4.7).

We conclude by mentioning some directions for future research. We have only solved the case $\beta=2$ of broken time-reversal symmetry. In that case the DMPK equation (1.5) can be mapped onto a free-fermion problem. For $\beta=1,4$ the Sutherland-type mapping which we have considered is onto an interacting Schrödinger equation. It might be possible to solve this equation exactly too, using techniques developed recently for the Sutherland Hamiltonian. ${ }^{29,30}$ From the work of Chalker and Macêdo $^{27}$ we know that the two-point correlation function in the large- $N$ limit has a simple $1 / \beta$ dependence on the symmetry index. This poses strong restrictions on a 
possible $\beta$ dependence of the eigenvalue interaction.

Another technical challenge is to compute the exact two-point correlation function $K\left(x, x^{\prime}, s\right)$ from the distribution function $P\left(\left\{x_{n}\right\}, s\right)$. Our result (3.5) for $P$ is exact, but the large- $N$ asymptotic result (3.22) for $K$ ignores fine structure on the scale of the eigenvalue spacing. (This large- $N$ result for $K$ corresponds to the regime of validity of the diagrammatic perturbation theory of UCF, ${ }^{6,7}$ while the exact result for $P$ goes beyond perturbation theory.) In RMT there exists a technique known as the method of orthogonal polynomials, ${ }^{9}$ which permits an exact computation of $K{ }^{39}$ A logarithmic interaction seems essential for this method to work, and we see no obvious way to generalize it to the nonlogarithmic interaction (5.1).

It might be possible to come up with another maximum-entropy principle, different from that of Muttalib, Pichard, and Stone, ${ }^{2}$ which yields the correct eigenvalue interaction (5.1) instead of the logarithmic interaction (1.2b). Slevin and Nagao ${ }^{40}$ have recently proposed an alternative maximum-entropy principle, but their dis- tribution function does not improve the agreement with Eq. (1.4). ${ }^{41}$

To go beyond quasi-one-dimensional geometries (long and narrow wires) remains an outstanding problem. A numerical study of Slevin, Pichard, and Muttalib ${ }^{42}$ has indicated a significant breakdown of the logarithmic repulsion for two- and three-dimensional geometries (squares and cubes). A generalization of the DMPK equation (1.5) to higher dimensions has been the subject of some recent investigations. ${ }^{43,44}$ It remains to be seen whether the method developed here for Eq. (1.5) is of use for that problem.

\section{ACKNOWLEDGMENTS}

This research was supported in part by the "Nederlandse organisatie voor Wetenschappelijk Onderzoek" (NWO) and by the "Stichting voor Fundamenteel Onderzoek der Materie" (FOM).
${ }^{1}$ Y. Imry, Europhys. Lett. 1, 249 (1986).

${ }^{2}$ K. A. Muttalib, J.-L. Pichard, and A. D. Stone, Phys. Rev. Lett. 59, 2475 (1987).

${ }^{3}$ A. D. Stone, P. A. Mello, K. A. Muttalib, and J.-L. Pichard, in Mesoscopic Phenomena in Solids, edited by B. L. Al'tshuler, P. A. Lee, and R. A. Webb (North-Holland, Amsterdam, 1991).

${ }^{4}$ P. A. Mello and J.-L. Pichard, Phys. Rev. B 40, 5276 (1989).

${ }^{5}$ C. W. J. Beenakker, Phys. Rev. Lett. 70, 1155 (1993); Phys. Rev. B 47, 15763 (1993).

${ }^{6}$ B. L. Al'tshuler, Pis'ma Zh. Eksp. Teor. Fiz. 41, 530 (1985) [JETP Lett. 41, 648 (1985)].

${ }^{7}$ P. A. Lee and A. D. Stone, Phys. Rev. Lett. 55, 1622 (1985); P. A. Lee, A. D. Stone, and H. Fukuyama, Phys. Rev. B 35, 1039 (1987).

${ }^{8}$ C. W. J. Beenakker and B. Rejaei, Phys. Rev. Lett. 71 , 3689 (1993).

${ }^{9}$ M. L. Mehta, Random Matrices, 2nd ed. (Academic, New York, 1991).

${ }^{10}$ K. B. Efetov, Adv. Phys. 32, 53 (1983).

${ }^{11}$ B. L. Al'tshuler and B. I. Shklovskiì, Zh. Eksp. Teor. Fiz. 91, 220 (1986) [Sov. Phys. JETP 64, 127 (1986)].

${ }^{12}$ R. A. Jalabert, J.-L. Pichard, and C. W. J. Beenakker, Europhys. Lett. 24, 1 (1993).

${ }^{13} \mathrm{~J}$.-L. Pichard, in Quantum Coherence in Mesoscopic Systems, Vol. 254 of NATO Advanced Study Institute, Series B: Physics, edited by B. Kramer (Plenum, New York, 1991).

${ }^{14}$ O. N. Dorokhov, Pis'ma Zh. Eksp. Teor. Fiz. 36, 259 (1982) [JETP Lett. 36, 318 (1982)].

15 P. A. Mello, P. Pereyra, and N. Kumar, Ann. Phys. (N.Y.) 181, 290 (1988).

${ }^{16}$ P. A. Mello and A. D. Stone, Phys. Rev. B 44, 3559 (1991).

${ }^{17}$ A. M. S. Macêdo and J. T. Chalker, Phys. Rev. B 46, 14985 (1992).

${ }^{18}$ A. Hüffmann, J. Phys. A 23, 5733 (1990).
${ }^{19}$ S. Iida, H. A. Weidenmüller, and J. A. Zuk, Phys. Rev. Lett. 64, 583 (1990).

${ }^{20}$ M. R. Zirnbauer, Phys. Rev. Lett. 69, 1584 (1992).

${ }^{21}$ P. A. Mello and B. Shapiro, Phys. Rev. B 37, 5860 (1988).

22 V. I. Mel'nikov, Fiz. Tverd. Tela 23, 782 (1981) [Sov. Phys. Solid State 23, 444 (1981)].

${ }^{23}$ P. A. Mello, J. Math. Phys. 27, 2876 (1986).

${ }^{24}$ P. A. Mello, Phys. Rev. Lett. 60, 1089 (1988).

${ }^{25}$ M. J. M. de Jong and C. W. J. Beenakker, Phys. Rev. B 46, 13400 (1992).

${ }^{26}$ C. W. J. Beenakker, Phys. Rev. B 49, 2205 (1994).

27 J. T. Chalker and A. M. S. Macêdo, Phys. Rev. Lett. 71, 3693 (1993).

${ }^{28}$ B. Sutherland, Phys. Rev. A 5, 1372 (1972).

${ }^{29}$ B. D. Simons, P. A. Lee, and B. L. Al'tshuler, Phys. Rev. Lett. 70, 4122 (1993).

${ }^{30}$ E. R. Mucciolo, B. S. Shastry, B. D. Simons, and B. L. Al'tshuler (unpublished).

${ }^{31}$ C. W. J. Beenakker and B. Rejaei, Physica A (to be published).

${ }^{32}$ Higher Transcendental Functions, edited by A. Erdélyi (McGraw-Hill, New York, 1953), Vol. 1, p. 175.

${ }^{33}$ The useful asymptotic relation (3.1) between a Legendre function and a Bessel function does not appear in the handbooks on special functions which we consulted. We obtained it by combining the two asymptotic expansions (8.10.7) and (9.2.1) in the Handbook of Mathematical Functions, edited by M. Abramowitz and I. A. Stegun (Dover, New York, 1972).

${ }^{34}$ F. J. Dyson, J. Math. Phys. 13, 90 (1972). Dyson's derivation of Eq. (3.8) is for a logarithmic interaction potential, but it is readily generalized to other repulsive interactions $u\left(x, x^{\prime}\right)$.

${ }^{35}$ M. Büttiker, Phys. Rev. Lett. 65, 2901 (1990).

${ }^{36}$ C. W. J. Beenakker, Phys. Rev. B 46, 12841 (1992).

${ }^{37}$ C. W. J. Beenakker, Phys. Rev. Lett. 67, 3836 (1991); 68, 1442(E) (1992). 
${ }^{38}$ C. W. J. Beenakker, in Transport Phenomena in Mesoscopic Systems, edited by H. Fukuyama and T. Ando (Springer, Berlin, 1992).

${ }^{39}$ K. A. Muttalib, Y. Chen, M. E. H. Ismail, and V. N. Nicopoulos, Phys. Rev. Lett. 71, 471 (1993).

${ }^{40}$ K. Slevin and T. Nagao, Phys. Rev. Lett. 70, 635 (1993).

${ }^{41}$ The distribution function of Ref. 40 yields $\operatorname{Var} G / G_{0}=$ $0.148 \beta^{-1}$, which is more than $10 \%$ above Eq. (1.4) [K.
Slevin (private communication)].

${ }^{42}$ K. Slevin, J.-L. Pichard, and K. A. Muttalib, J. Phys. I (France) 3, 1387 (1993).

${ }^{43}$ P. A. Mello and S. Tomsovic, Phys. Rev. Lett. 67, 342 (1991).

44 J. T. Chalker and M. Bernhardt, Phys. Rev. Lett. 70, 982 (1993). 